\title{
PREPARATION AND CHARACTERIZATION OF PLGA NANOPARTICLES \\ BY
}

Dina O. Helal, Amany O. Kamel, Gehanne A.S. Awad, Ahmed S. Geneidi. FROM

Faculty of Pharmacy, Department of Pharmaceutics and Industrial Pharmacy, Ain Shams University, Cairo, Egypt

\begin{abstract}
Nanoparticles were prepared by emulsion solvent evaporation method, using methylene chloride (DCM) as the organic solvent and poly vinyl alcohol (PVA) as the surfactant. Different formulation conditions such as surfactant concentration, drug amount, homogenization time, aqueous phase volume were investigated for drug entrapment efficiency, drug loading and particle size. It was found that PVA concentration less than $1 \%$ was unable to form a stable emulsion, increasing drug amount more than $30 \mathrm{mg}$ didn't lead to any significant difference in drug entrapment efficiency or particle size, homogenization for 2 minutes led to particles having larger size and higher entrapment efficiency than those obtained after 3 minutes homogenization, increasing the aqueous phase volume from $40 \mathrm{ml}$ to $80 \mathrm{ml}$ led to a significant increase in entrapment efficiency and a significant decrease in particle size.

\section{Introduction}

Nanotechnology is the science involved in the design, synthesis, characterization and application of materials and devices whose smallest functional organization is on the nanometer scale at least on one dimension (Sahoo and Labhasetwar 2003).

Nanocarriers can be made from a variety of materials such as lipids (liposomes, solid lipid nanoparticlers), polymers (nano/microparticles, micelles, dendrimers), inorganic carriers (gold nanoparticles, carbon spheres and nanotubes) as shown in Figure I According to the manufacturing methods and materials used, these carriers may have different shapes and sizes with distinct properties(Qiu and Bae 2006). 


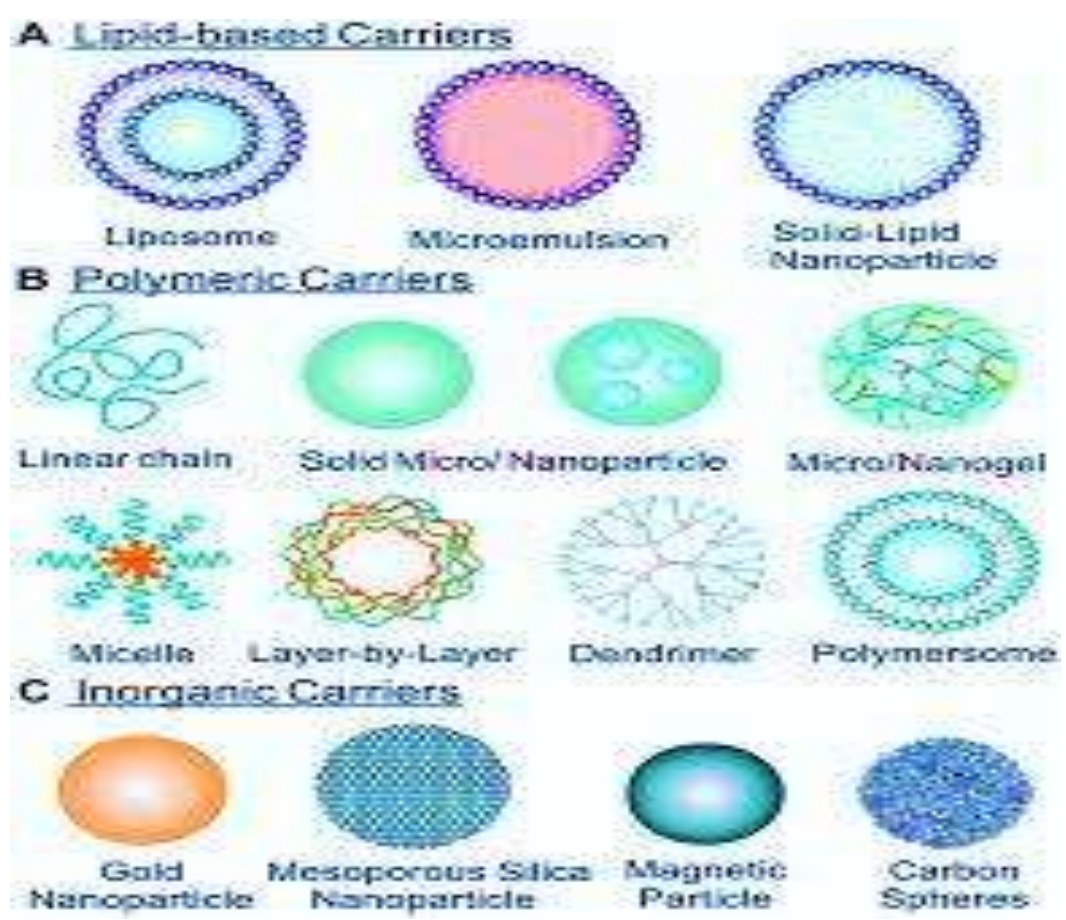

Figure I : Schematic illustration of different micro/nano carriers used for drug delivery (Mo et al. 2014).

Polymeric NPs are considered a versatile medium for the delivery and monitoring of highly toxic compounds in vivo due to their enhanced biological stability and extended in vivo circulation. They account for more than $80 \%$ of the available therapeutics in clinical use (Ravi Kumar 2000) due to their small particle size which facilitates their penetration into smaller capillaries and their subsequent uptake by the cells, which allows efficient drug accumulation at the target sites. Also, being synthesized from biodegradable materials allows sustained drug release within the target site over a period of days or even weeks (Sahoo \& Labhasetwar 2003).

Both synthetic and natural polymers have an important role as biomedical materials for drug delivery, yet synthetic biodegradable polymers have been increasingly used to deliver drugs. Among the synthetic polymers, the thermoplastic aliphatic poly(esters) such as polylactic acid(PLA), polyglycolic acid (PGA) and, especially PLGA, have generated tremendous interest due to their excellent biocompatibility, biodegradability and toxicologically safe by-products (Acharya and Sahoo 2011).

PLGA is a copolymer of lactic acid and glycolic acid. Depending on the used ratio of lactide to glycolide, different forms of PLGA can be obtained, which are usually identified with regard to the monomers ratio used (e.g., PLGA 75:25 identifies a copolymer whose composition is $75 \%$ lactic acid and $25 \%$ glycolic acid). PLGA is one of the most successfully used biodegradable polymers for the development of nanomedicines because it undergoes hydrolysis in the body to produce the biodegradable metabolite monomers, lactic acid and glycolic acid (Figure II) which are endogenous 
and easily metabolized by the body via kreb's cycle thus resulting in minimal systemic toxicity (Acharya \& Sahoo 2011; Kumari et al 2010). 
The rate of degradation of PLGA depends on the molar ratio of lactic and glycolic acids in the polymer chain, molecular weight of the polymer, degree of crystallinity and the glass transition temperature $(\mathrm{Tg})$ of the polymer. By manipulating the molecular weight and lactide/glycolide ratio, the degradation time of PLGA and, subsequently, the release profile can be varied accordingly (Lu et al. 2009).

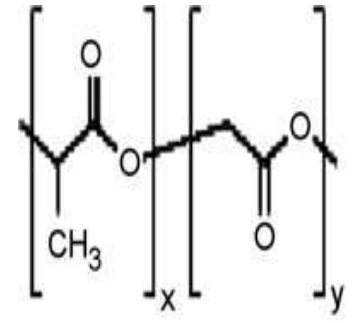

PLGA
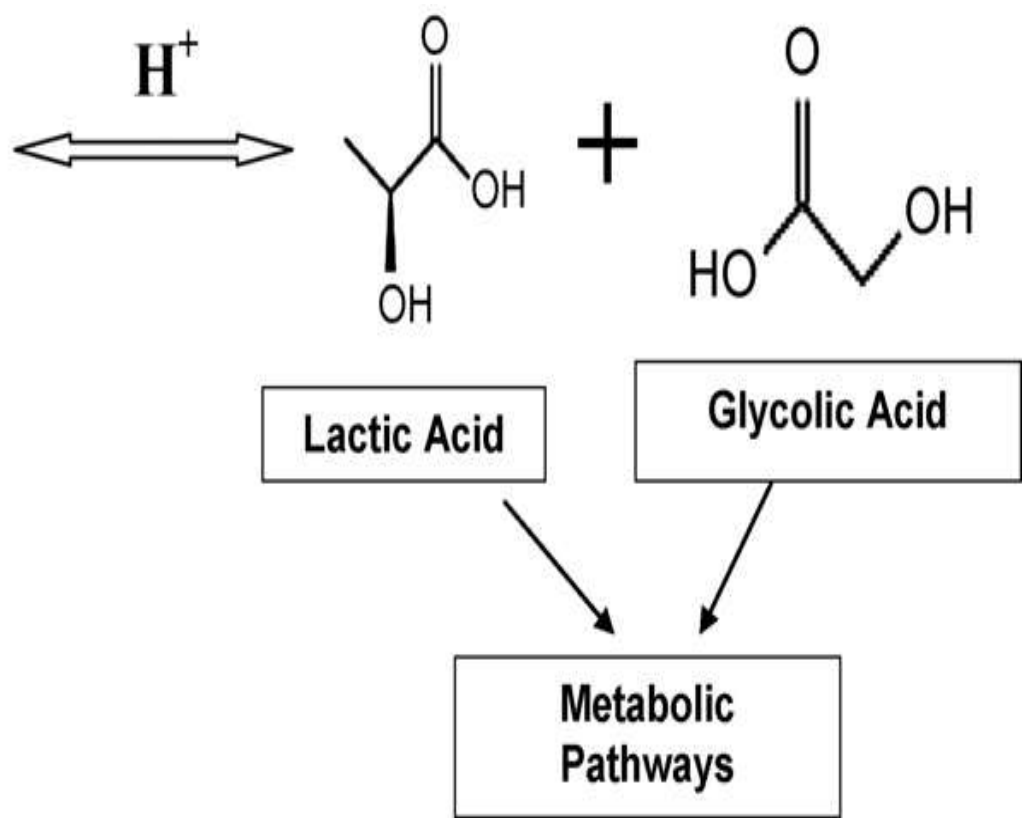

Figure II: Illustration of PLGA structure and its endogenous degradation products

A wide variety of therapeutic agents, including low molecular weight lipophilic or hydrophilic drugs, high molecular weight DNA or antisense DNA can be encapsulated inside NPs. They can be formulated for targeted delivery to the lymphatic system, brain, arterial walls, lungs, liver, spleen, or made for long-term systemic circulation. The entrapped moiety in the polymeric matrix of NPs is released at a sustained rate by diffusion or by degradation of the polymeric matrix, thereby giving a sustained release formulation to elicit enhanced therapeutic efficacy (Parveen and Sahoo 2008).

According to the nature of the therapeutic agent intended to be entrapped within the PLGA nanoparticles and the desired delivery route, many approaches are proposed for preparing the nanoparticles as shown in Figure III (Hans and Lowman 2002;Kumari et al 2010) such as Double/ multiple emulsion method, Solvent diffusion method, Nano-precipitation method and emulsion solvent evaporation method which is the most commonly used method for the preparation of polymeric nanoparticles. It has been successful for encapsulating hydrophobic drugs, but has poor results incorporating bioactive agents of a hydrophilic nature. 


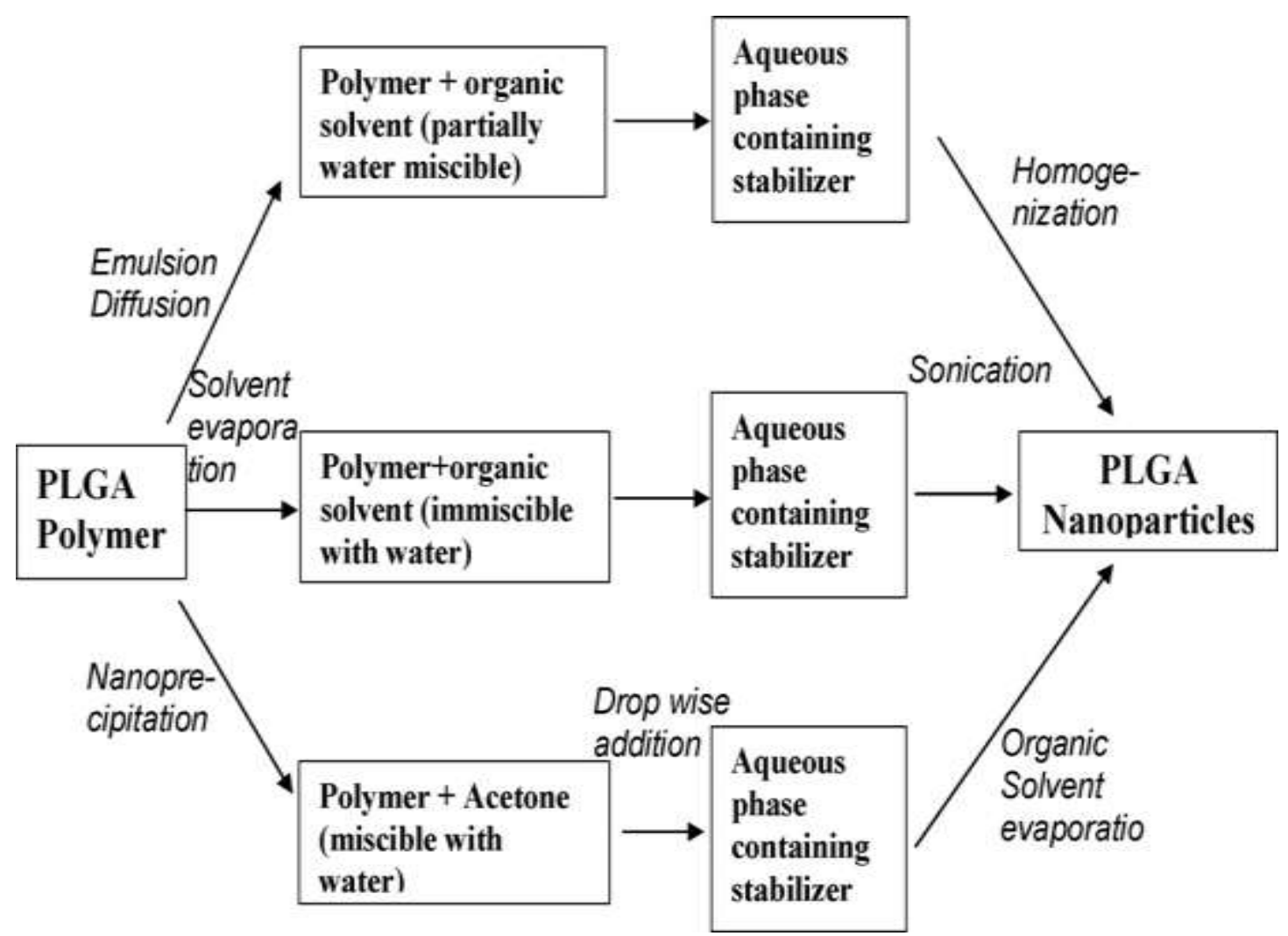

Figure III: Different methods of PLGA nanoparticles preparation (Kumari et al 2010)

The aim of this work is to prepare PLGA nanoparticles loaded with a model hydrophobic drug using the solvent evaporation method. The effect of different formulation variables such as: surfactant concentration, homogenization time, amount of drug, volume of aqueous phase, on the obtained NPs' characteristics will be assessed

\section{Materials and methods}

\section{Materials}

Dichloro methane (DCM),El Nasr pharmaceutical company, Cairo, Egypt.Dimethyl Sulfoxide (DMSO), sigma- Aldrich, USA. PDLG DLlactide/Glycolidecopolymer:Lactide/Glycolide ratio 50:50 with intrinsic viscosity 0.4 dl/g (PLGA 5004) was kindly supplied by Purac biomaterials, Netherlands.Poly vinyl alcohol (PVA), Sigma- Aldrich, USA.

\section{Preparation of PLGA nanoparticles}

A certain amount of the drug and PLGA were dissolved in different amounts of dichloromethane (DCM) and sonicated in a bath ultrasonicator for 3 minutes to ensure complete dissolution of the drug and the polymer. This solution was then added portionwise to Poly vinyl alcohol (PVA) solution to form an o/w emulsion which was broken down into nanodroplets by homogenization at $17900 \mathrm{rpm}$. The formed emulsion was then transferred to a magnetic stirrer and stirred till complete evaporation of the 
organic phase under atmospheric conditions to form the colloidal suspension of PLGA nanoparticles (NPs). Accurately measured amounts of NP suspension were centrifuged at $15000 \mathrm{rpm}$ for 15 minutes at $4^{\circ} \mathrm{C}$, then washed twice with distilled water. The pellets were then collected for further characterization. All nanoparticle suspensions were prepared in triplicate (Budhian et al. 2007;Mu and Feng 2003;Pietzonka et al. 2002; Yin Win and Feng 2005)

\section{Experimental design}

Alterations were made to the formulation conditions of nanoparticles including PVA concentration in the external phase, amount of drug, homogenization time and aqueous phase volume to find out the optimal conditions for drug entrapment and drug loading (table 1, 2). All trials were done in triplicate using 200 mg PLGA.

\section{Characterization of the prepared PLGA nanoparticles}

\section{Particle morphology}

A sample of the prepared NPs was placed on a microscope slide, diluted with distilled water and evaluated morphologically using optical microscope (Axiostar plus, Zeiss, USA)

\section{Determination of the drug encapsulation efficiency (EE\%) and Drug Loading} (DL \%)

Definite amount of the sedimented NPs were dissolved in $1 \mathrm{ml} \mathrm{DMSO}$ and sonicated in a bath ultrasonicator for 2 minutes to ensure complete dissolution of all NPs which was then assayed spectrophotometrically against a blank formula using Spectrophotometer (Shimadzu, Tokyo, Japan). All measurements were done in triplicate(Joshi et al. 2010) and the encapsulation efficiency EE (\%)was calculated according to the following equation

$$
\mathrm{EE}(\%)=\frac{\text { Amount of drug in NPS } X 100}{\text { Amount of drug used in formulation }} \quad \text { Equation (1) }
$$

The drug loading (DL) was calculated according to the following equation

$$
\mathrm{DL}(\%)=\frac{\text { Amount of drug in NPS } \times 100}{\text { Amount of drug in NPs +amount of polymer used in formula }} \quad \text { Equation (2) }
$$


Table (1): Factors and levels used for optimization of NPs preparation conditions

\begin{tabular}{|l|l|}
\hline Factor & Level \\
\hline & $0.25 \%$ \\
PVA \% & $0.5 \%$ \\
& $1 \%$ \\
& \\
\hline & $10 \mathrm{mg}$ \\
Drug amount & $30 \mathrm{mg}$ \\
& $40 \mathrm{mg}$ \\
\hline Homogenization time & $2 \mathrm{minutes}$ \\
& $3 \mathrm{minutes}$ \\
\hline & $40 \mathrm{ml}$ \\
Aqueous phase volume & $60 \mathrm{ml}$ \\
& $80 \mathrm{ml}$ \\
\hline
\end{tabular}

Particle size and size distribution determination of NPs.

The NPs were suspended in distilled water and the average particle diameter (Zaverage), and size distribution (PDI) of NPs were measured by dynamic light scattering using Particle size analyzer (Malvern Zetasizer, Malvern Instruments Ltd., Malvern, UK). The reported results are the mean of three determinations.

Table 2: Composition of NPs formulations to study the effect of changing various formulation conditions

\begin{tabular}{|c|c|c|c|c|}
\hline Formula & PVA \% & Homogenization time & Drug amount & Aqueous phase \\
\hline F1 & 0.25 & 3 & 30 & 80 \\
\hline F2 & 0.5 & 3 & 30 & 80 \\
\hline F3 & 1 & 3 & 30 & 80 \\
\hline F4 & 1 & 2 & 30 & 80 \\
\hline F5 & 1 & 3 & 10 & 80 \\
\hline F6 & 1 & 3 & 40 & 80 \\
\hline F7 & 1 & 3 & 30 & 40 \\
\hline F8 & 1 & 3 & 30 & 60 \\
\hline
\end{tabular}




\section{Zeta potential determination.}

The NPs were suspended in deionized water and the Zeta potential of NPs was measured using a Malvern Zetasizer 4. The results obtained are a mean of three determinations.

\section{Statistical analysis}

All statistical analysis were undertaken using instat program

\section{Results}

Table (3): Effect of various formulation conditions on the EE\%, DL\%, particle size, PDI and zeta potential of NPs

\begin{tabular}{|c|c|c|c|c|c|}
\hline Formula & EE\% \pm SD & DL\% \pm SD & $\begin{array}{c}\text { Particle size } \\
(\mathbf{n m}) \pm \text { SD }\end{array}$ & PDI \pm SD & $\begin{array}{c}\text { Zeta potential } \\
\text { (mv) }\end{array}$ \\
\hline F1 & \multicolumn{5}{|c|}{ Unstable Emulsion } \\
\hline F2 & \multicolumn{5}{|c|}{ Unstable Emulsion } \\
\hline F3 & $37.19 \pm 0.74$ & $5.28 \pm 0.1$ & $329.2 \pm 5.17$ & $0.33 \pm 0.04$ & $-6.55 \pm 0.046$ \\
\hline F4 & $12.27 \pm 1.92$ & $0.6 \pm 0.09$ & $325.5 \pm 16.76$ & $0.35 \pm 0.08$ & $-6.89 \pm 0.43$ \\
\hline F5 & $25.43 \pm 1.1$ & $4.83 \pm 0.2$ & $334.6 \pm 7.7$ & $0.443 \pm 0.054$ & $-7.44 \pm 0.82$ \\
\hline F6 & $44.61 \pm 1.02$ & $6.26 \pm 0.13$ & $677.2 \pm 21.98$ & $0.65 \pm 0.075$ & $-7.73 \pm 0.101$ \\
\hline F7 & $20.13 \pm 2.5$ & $2.93 \pm 0.35$ & $372.3 \pm 7.07$ & $0.24 \pm 0.06$ & $-8.12 \pm 0.21$ \\
\hline F8 & $28.66 \pm 1.49$ & $4.12 \pm 0.2$ & $381.9 \pm 1.21$ & $0.3 \pm 0.04$ & $-7.43 \pm 0.246$ \\
\hline
\end{tabular}

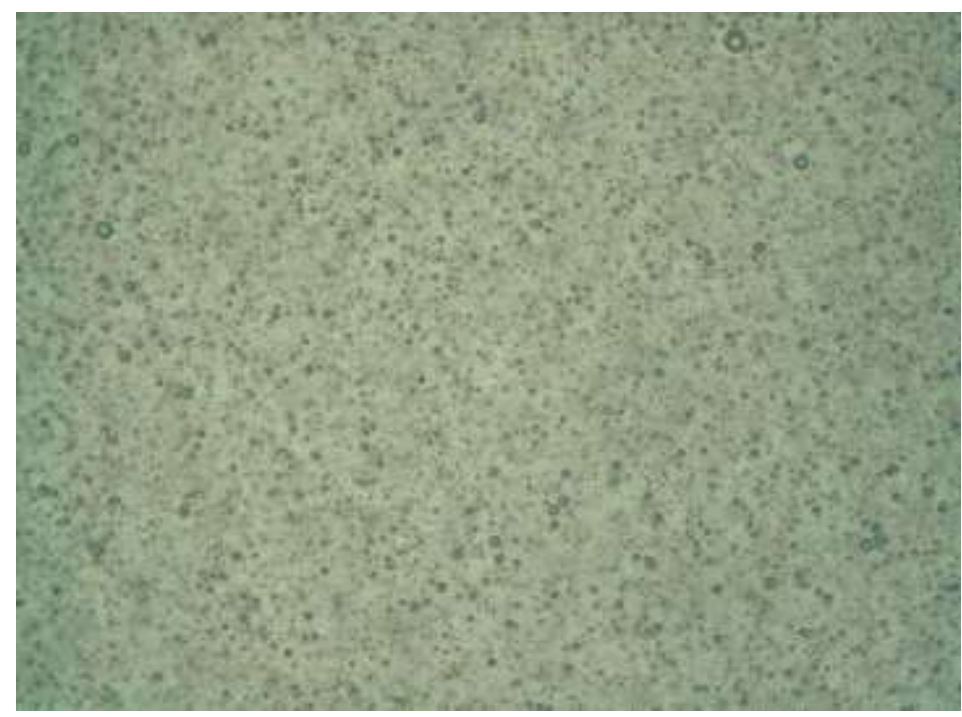

Figure (1): Photomicrograph of NPs prepared using PLGA 5004 


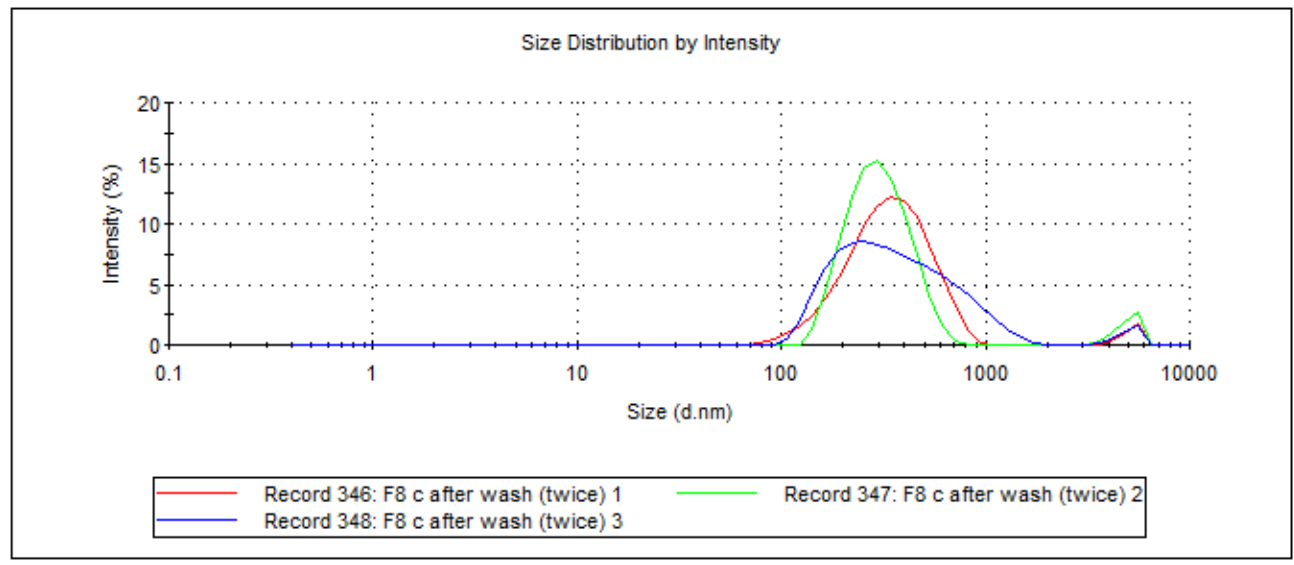

Figure (2) : z-avg chart representation for F3

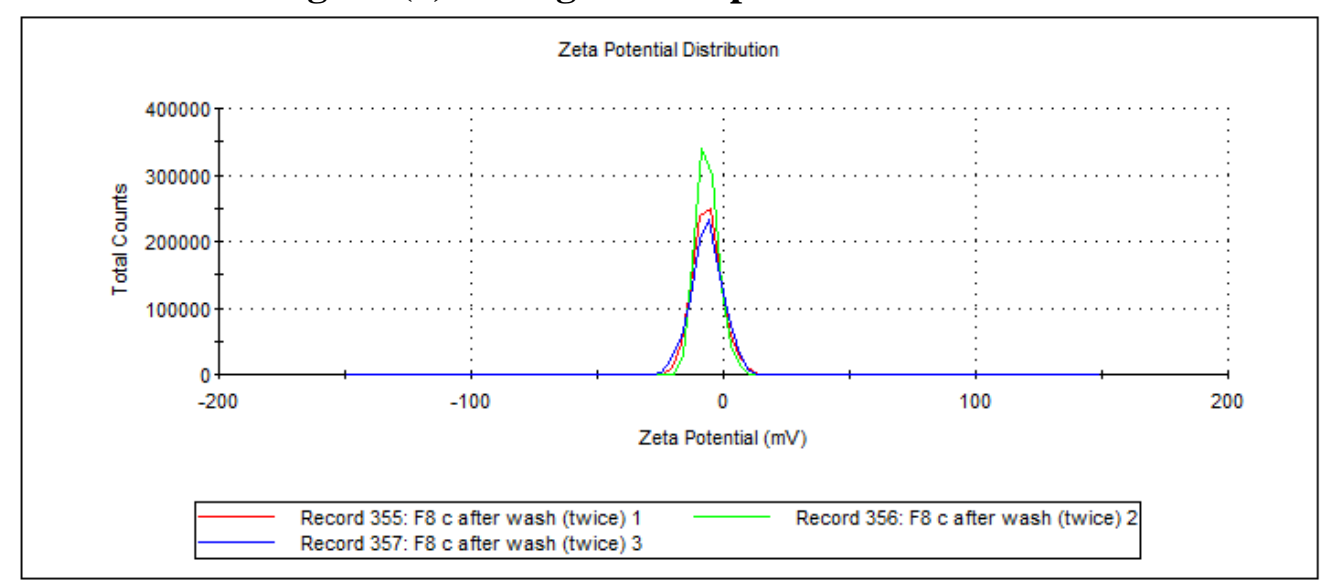

Figure (3): Zeta potential chart for F3 

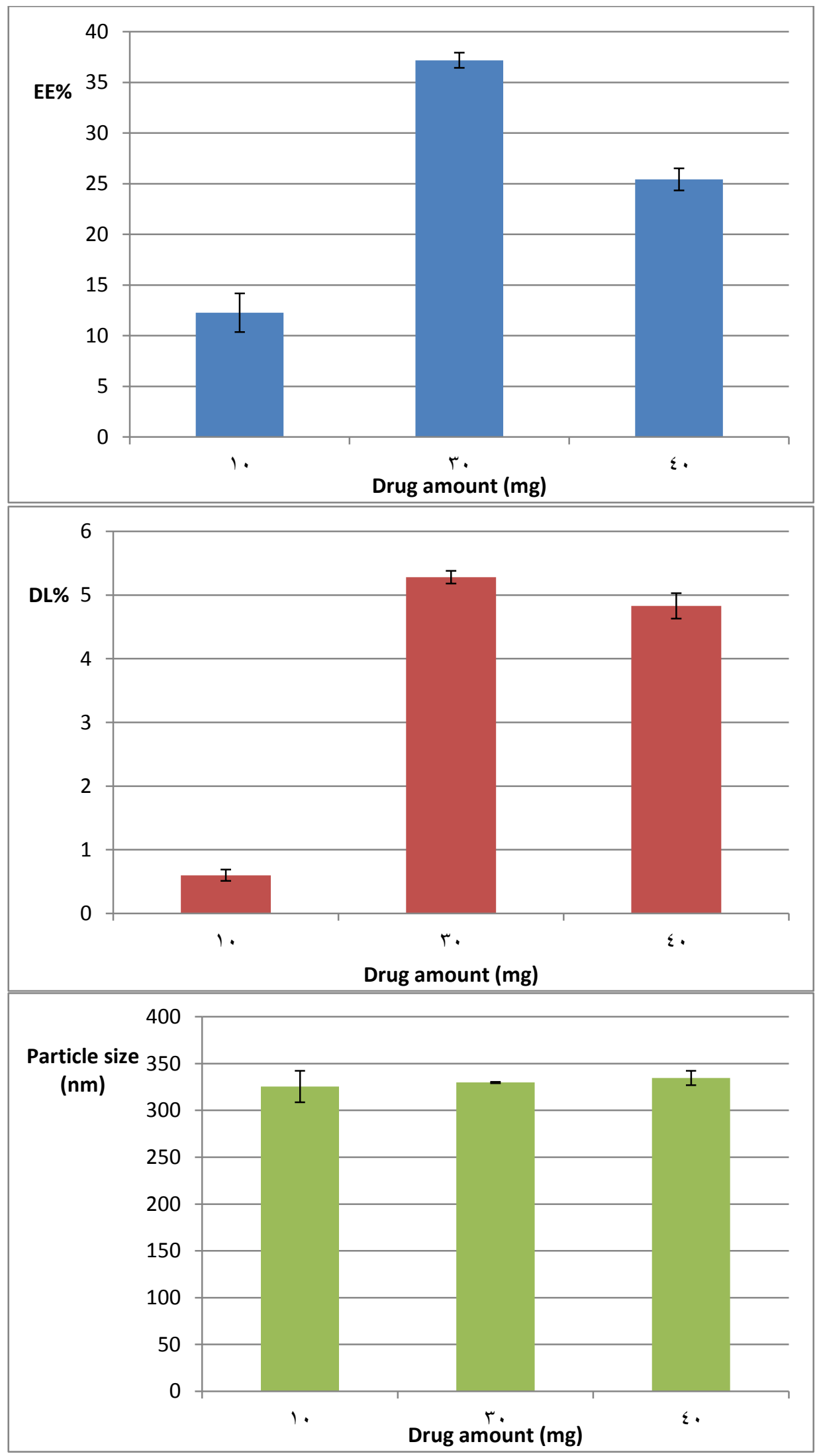

Figure (4): Bar chart showing the effect of drug amount on (a) entrapment efficiency \%, (b) drug loading \%, (c) particle size (nm) 


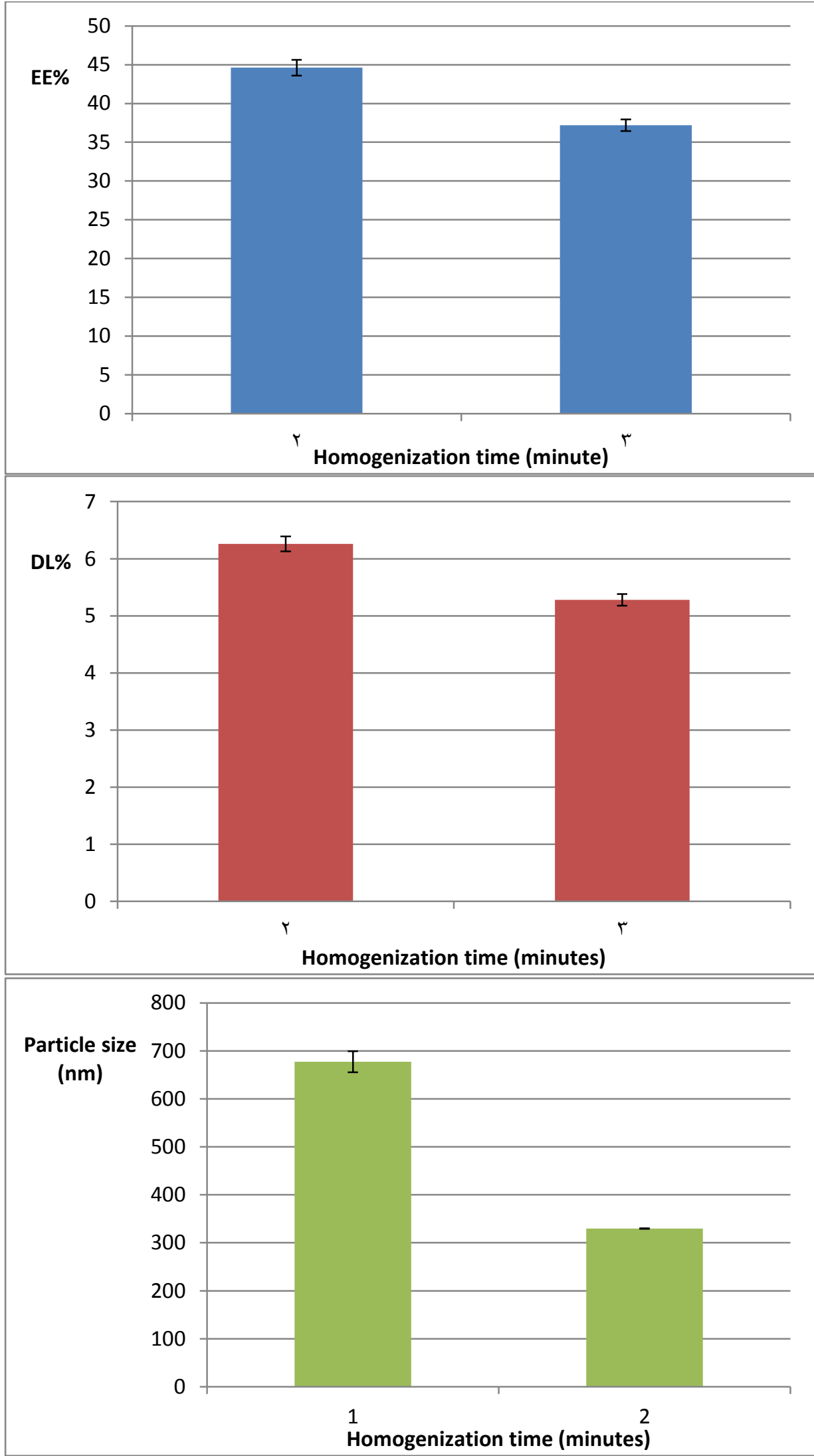

Figure (5): Bar chart showing the effect of homogenization time on (a) entrapment efficiency \%, (b) drug loading \%, (c) particle size (nm) 

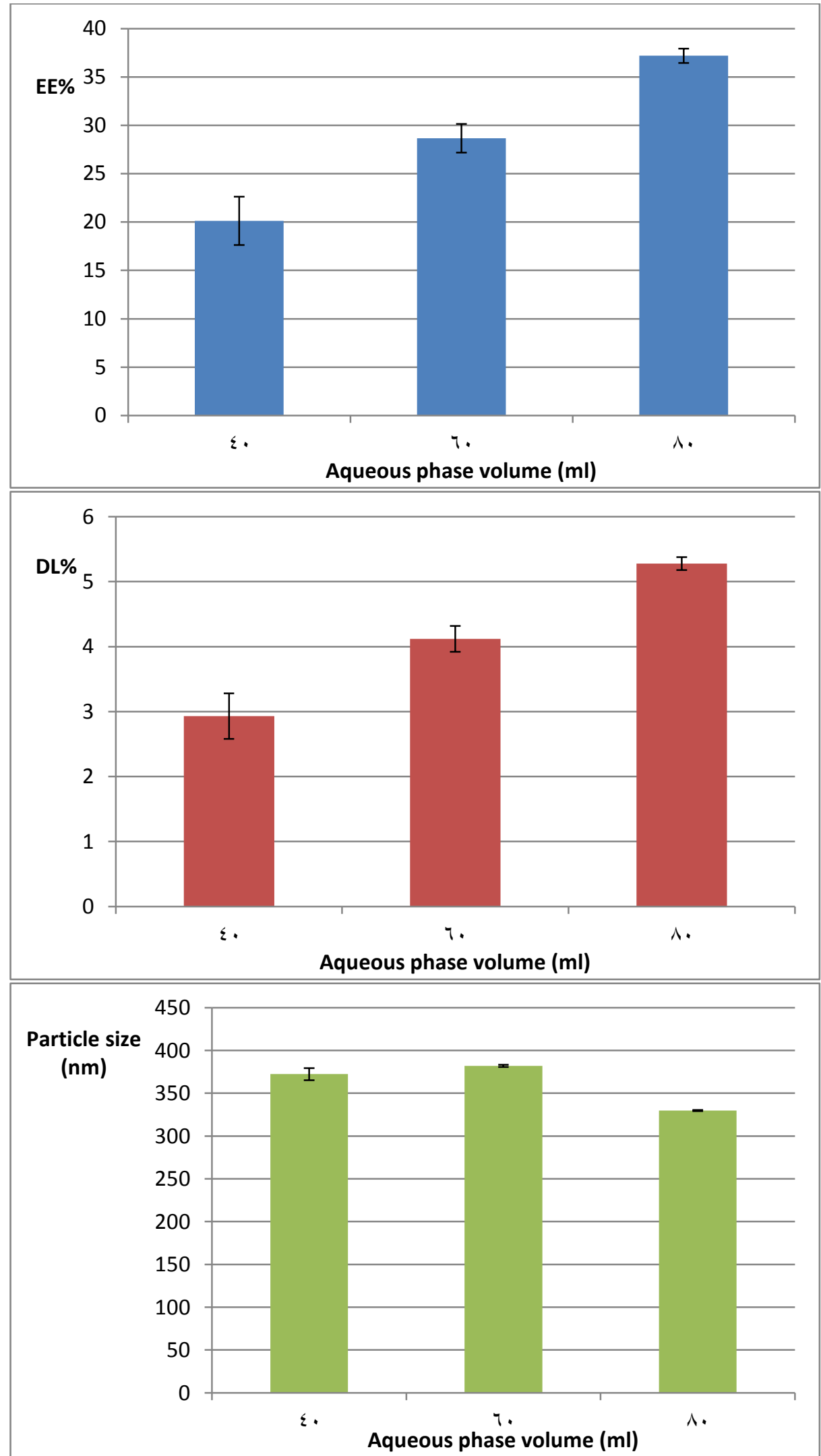

Figure (6): Bar chart showing the effect of aqueous phase volume on (a) entrapment efficiency \%, (b) drug loading \%, (c) particle size (nm) 


\section{Discussion}

\section{Preparation of PLGA nanoparticles}

Emulsion solvent evaporation is the most common method used for the encapsulation of hydrophobic drugs where both the drug and the polymer are dissolved in an organic phase which is then emulsified with the aqueous phase. The emulsion is converted into NP suspension by diffusion of the polymer organic solvent into the external aqueous phase and its subsequent evaporation either under reduced pressure or at atmospheric pressure (Vauthier and Bouchemal 2009;Zhang et al. 2013).

The prepared NPs showed uniformly shaped spherical particles in the nanometer range as shown by photo microscopic analysis in figure 1.

Zeta potential is an important factor which ensures the particle stability with aging where the repulsive forces prevent aggregation of particles, the zeta potential obtained for the prepared NPs was found to be in the range of -6.39 to $-8.12 \mathrm{mv}$ (table 3). This was expected due to the interaction of the cationic drug with the carboxylic groups of the polymer on the surface of NPs thus leading to a decrease in the surface charge of drug loaded NPs. The same finding was previously observed with (ZakeriMilani et al. 2013) in Vancomycin PLGA NPs. Also the use of PVA as an emulsifier during NPs preparation contributes to the obtained low values of zeta potential, where a fraction of the PVA used forms a stable network on the surface of NPs which can't be removed by washing, and so this layer of residual PVA shields the surface charge of PLGA (Manchanda et al. 2010;Sahoo et al. 2002).

\section{Effect of different preparation conditions on the NPs characteristics}

\section{a) PVA concentration}

Polyvinyl alcohol (PVA) is the most commonly used emulsifier for the preparation of PLGA nanoparticles because the particles obtained using this emulsifier are relatively uniform, smaller in size, and can be easily re-dispersed in aqueous medium (Panyam and Labhasetwar 2012).

Different concentrations of PVA were tested to determine the most suitable concentration for NPs preparation, both 0.25 and $0.5 \%$ PVA gave unstable emulsions, while 1\% PVA gave a stable o/w emulsion with EE\% $37.19 \pm 0.74$, DL\% $5.28 \pm 0.1$, Particles size $329.2 \pm 5.17 \mathrm{~nm}$ and PDI $0.33 \pm 0.04$ (table 3).

\section{b) Drug amount}

Different drug amounts $(10,30,40 \mathrm{mg})$ were tested. As shown in table 3, figure 4, the entrapment efficiency for $10 \mathrm{mg}$ drug was very low $(12.27 \pm 1.92 \%)$ due to the very small amount of drug used relative to the large volume of the aqueous phase which might have led to the escape of the drug into the aqueous phase. Increasing the drug amount from $30 \mathrm{mg}$ to $40 \mathrm{mg}$ led to a significant decrease in the entrapment efficiency $(\mathrm{P}<0.0001)$ of the drug $(25.43 \pm 1.1 \%, 37.19 \pm 0.74 \%)$ and drug loading $(\mathrm{P}<0.05)$ of the prepared NPs $(4.83 \pm 0.1 \%, 5.28 \pm 0.2 \%)$ for the $40,30 \mathrm{mg}$ drug, respectively. This shows that $30 \mathrm{mg}$ is the optimum amount of drug to be entrapped since any increase in the used amount of drug doesn't increase the drug loading. There was no significant 
difference $(\mathrm{P}>0.05)$ in the particle size obtained by the 3 amounts of drug $(325.5 \pm$ $16.76,329.2 \pm 5.17,334.6 \pm 7.7 \mathrm{~nm}$ ) for $10,30,40 \mathrm{mg}$, respectively.

\section{c) Homogenization time}

After portion-wise addition of the organic phase to the aqueous phase, the emulsion was homogenized at $17900 \mathrm{rpm}$ for 2 minutes, and the particle size of the nanoparticles obtained was found to be $677.2 \pm 21.98 \mathrm{~nm}$, PDI $0.655 \pm 0.075$, while after homogenization for 3 minutes, the particle size obtained was $329.2 \pm 5.17 \mathrm{~nm}$, with PDI $0.31 \pm 0.04$ (table 3, figure 5). Due to the better particle size and it's more uniform distribution, 3 minute homogenization was selected for further study.

The EE\% after 2 minutes homogenization was $44.61 \pm 1.02 \%$ which was significantly higher $(\mathrm{p}<0.05)$ than the EE\% $37.19 \pm 0.74 \%$ obtained after 3 minutes homogenization because of the larger particle size obtained after 2 minutes homogenization which enables entrapment of a larger drug amount.

\section{d) Aqueous phase volume}

Three volumes of aqueous phase $(40,60,80 \mathrm{ml})$ were tested while maintaining a constant Organic : Aqueous phase volume ratio of 1:2 to evaluate the effect of the volume of aqueous phase on the EE\%, DL\% and particle size of NPs. A constant amount of drug $(30 \mathrm{mg})$ and a constant amount of polymer $(200 \mathrm{mg})$ were used. It was found that by increasing the volume of the aqueous phase there was a significant increase in the EE\% and DL\% ( $p<0.05$ ). The EE \% was $20.13 \pm 2.5,28.663 \pm 1.49$ and $37.19 \pm 0.745$, while the DL\% was $2.93 \pm 0.35,4.121 \pm 0.205$ and $5.283 \pm 0.1$ for the 40 , $60,80 \mathrm{ml}$ aqueous phase volume, respectively as shown in table 3 and figure 6.

Increasing the volume of the aqueous phase while maintaining a constant PLGA weight lead to a faster precipitation of the polymer thus leading to the preparation of less porous NPs which in turn have higher EE\% and DL\% than those prepared using less aqueous volume.(Mao et al. 2008).

It was found that the particle size of NPs prepared using 40,60, $80 \mathrm{ml}$ aqueous phase is $372.3 \pm 7.07,381.9 \pm 1.217$ and $329.2 \pm 5.17 \mathrm{~nm}$ respectively. There was an insignificant difference $(\mathrm{P}>0.05)$ in the particle size obtained using $40,60 \mathrm{ml}$ aqueous phase but further increase in the aqueous phase volume led to a decrease in the particle size where the organic phase volume was increased to maintain a constant organic : aqueous phase volume of $1: 2$ at a constant polymer weight. This leads to a lower polymer concentration and a subsequent decrease in the viscosity of the organic phase, thus increasing the net shear stress and promoting the formation of smaller particles, also decreasing the viscosity enhances the rapid dispersion of the internal phase into the external phase which leads to the formation of smaller droplets (Sansdrap and Moes. 1993; Wang.et al 2013).

\section{Conclusion}

The influence of several parameters on the preparation of PLGA nanoparticles by emulsion solvent evaporation was shown. In particular PVA concentration less than $1 \%$ was unable to form a stable emulsion, increasing drug amount more than $30 \mathrm{mg}$ 
didn't lead to any significant difference in drug entrapment efficiency or particle size, homogenization for 2 minutes led to particles having larger size $(677.2 \mathrm{~nm})$ and higher entrapment efficiency (44.61\%) than those obtained after 3 minutes homogenization $(329.2 \mathrm{~nm}),(37.19 \%)$, increasing the aqueous phase volume from $40 \mathrm{ml}$ to $80 \mathrm{ml}$ led to a significant increase in entrapment efficiency $(20.13,37.19 \%)$ and a significant decrease in particle size ( $372.3,329.2 \mathrm{~nm}$ ) for $40,80 \mathrm{ml}$ respectively, so the optimum formula can be prepared using 1\% PVA, $30 \mathrm{mg}$ drug, 3 minute homogenization and 80 $\mathrm{ml}$ aqueous phase.

\section{REFERENCES}

Acharya, S. \& Sahoo, S.K.(2011). PLGA nanoparticles containing various anticancer agents and tumour delivery by EPR effect. Advanced drug delivery reviews 63 ,(3) $170-183$

Budhian, A., Siegel, S.J., \& Winey, K.I. (2007). Haloperidol-loaded PLGA nanoparticles: Systematic study of particle size and drug content. International Journal of Pharmaceutics, 336, (2) 367-375

Hans, M.L. \& Lowman, A.M. (2002). Biodegradable nanoparticles for drug delivery and targeting. Current Opinion in Solid State and Materials Science, 6, (4) 319-327

Joshi, S.A., Chavhan, S.S., \& Sawant, K.K. (2010). Rivastigmine-loaded PLGA and PBCA nanoparticles: Preparation, optimization, characterization, in vitro and pharmacodynamic studies. European Journal of Pharmaceutics and Biopharmaceutics, 76, (2) 189-199

Kumari et al (2010). Biodegradable polymeric nanoparticles based drug delivery systems. Colloids and Surfaces B: Biointerfaces, 75, (1) 1-18

Lu ‘J.M., Wang, X.F., Marin-Muller, C.F., Wang, H.F., Lin PH FAU - Yao, Q., Yao, Q.F., \& Chen, C. (2009). Current advances in research and clinical applications of PLGA-based nanotechnology. Expert review of molecular diagnostics 9 , (4) 325-341

Manchanda, R., Fernandez-Fernandez, A., Nagesetti, A., \& McGoron, A.J. (2010). Preparation and characterization of a polymeric (PLGA) nanoparticulate drug delivery system with simultaneous incorporation of chemotherapeutic and thermo-optical agents. Colloids and Surfaces B: Biointerfaces, 75, (1) 260-267

Mao, S., Shi, Y., Li, L., Xu, J., Schaper, A., \& Kissel, T. (2008). Effects of process and formulation parameters on characteristics and internal morphology of poly(d,l-lactide-co-glycolide) microspheres formed by the solvent evaporation method. European Journal of Pharmaceutics and Biopharmaceutics, 68, (2) 214-223

Mo, R., Jiang, T.F., Di, J.F., Tai, W.F., \& Gu, Z. (2014). Emerging micro- and nanotechnology based synthetic approaches for insulin delivery. Chemical society reviews 43, (10) 3595-3629 
Mu, L \& .Feng, S.S. (2003). A novel controlled release formulation for the anticancer drug paclitaxel (Taxol_«): PLGA nanoparticles containing vitamin E TPGS. Journal of Controlled Release, 86, (1) 33-48

Panyam, J. \& Labhasetwar, V. (2012). Biodegradable nanoparticles for drug and gene delivery to cells and tissue. Advanced Drug Delivery Reviews, 64, Supplement, (0) 61-71

Parveen, S. \& Sahoo, S.K.( 2008). Polymeric nanoparticles for cancer therapy. Journal for drug targeting 16,(2) 108-123

Pietzonka, P., Walter, E., Duda-Johner, S., Langguth, P., \& Merkle, H.P. (2002). Compromised integrity of excised porcine intestinal epithelium obtained from the abattoir affects the outcome of in vitro particle uptake studies. European Journal of Pharmaceutical Sciences, 15, (1) 39-47

Qiu, L.Y. \& Bae, Y.H. (2006). Polymer architecture and drug delivery. Pharmaceutical research 23, (1) 1-30

Ravi Kumar, M.N. (2000). Nano and microparticles as controlled drug delivery devices. Journal of pharmacy and pharmaceutical sciences 3,(2) 234-258

Sahoo, S.K. \& Labhasetwar, V.(2003). Nanotech approaches to drug delivery and imaging. Drug Discovery Today, 8, (24) 1112-1120

Sahoo, S.K., Panyam, J., Prabha, S., \& Labhasetwar, V. (2002). Residual polyvinyl alcohol associated with poly (d,l-lactide-co-glycolide) nanoparticles affects their physical properties and cellular uptake. Journal of Controlled Release, 82, (1) $105-114$

Sansdrap \& Moes. (1993). Influence of manufacturing parameters on the size characteristics and the release profiles of nifedipine from poly(DL-lactide-coglycolide) microspheres. International Journal of Pharmaceutics, 98, (1-3) 157-164

Vauthier, C. \& Bouchemal, K. (2009). Methods for the preparation and manufacture of polymeric nanoparticles. Pharmaceutical research 26,(5) 1025-1058

Wang.et al (2013). Effect of preparation conditions on the size and encapsulation properties of mPEG-PLGA nanoparticles simultaneously loaded with vincristine sulfate and curcumin. Pharmaceutical development and technology 18, (3) 694-700

Yin Win, K. \& Feng, S.S. (2005). Effects of particle size and surface coating on cellular uptake of polymeric nanoparticles for oraldelivery of anticancer drugs. Biomaterials, 26,(15)2713-2722

Zakeri-Milani, P., Loveymi, B.D., Jelvehgari, M., \& Valizadeh, H. (2013). The characteristics and improved intestinal permeability of vancomycin PLGAnanoparticles as colloidal drug delivery system. Colloids and Surfaces B: Biointerfaces, 103, (0) 174-181 
Zhang, Y., Chan, H.F., \& Leong, K.W. (2013). Advanced materials and processing for drug delivery: The past and the future. Advanced Drug Delivery Reviews, 65, (1) 104-120

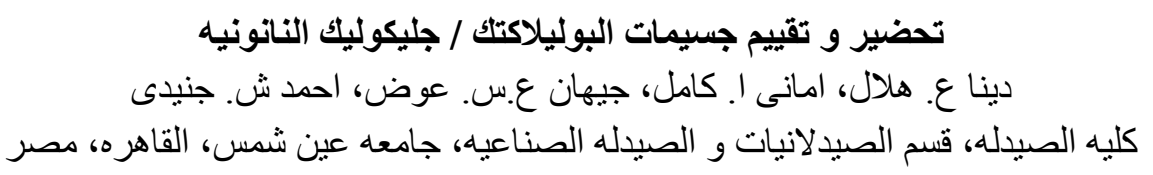

تم تحضير الجسيمات النانوية باستخدام طريقة تبخير مذيب المستحلب، وذلك باستخدام كلوريد الميثيلين

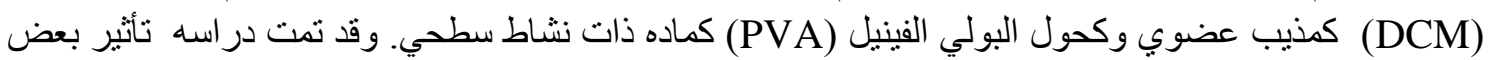
المتغير ات مثل تركيز الماده ذات النشاط السطحى، كمية العقار، وقت التجانس، وحجم الوسط الخارجي على التى كفاءة

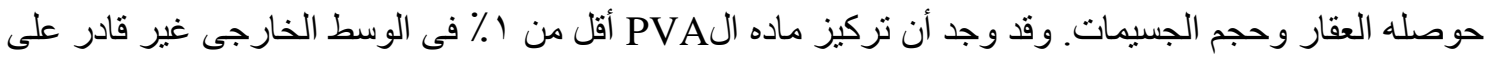

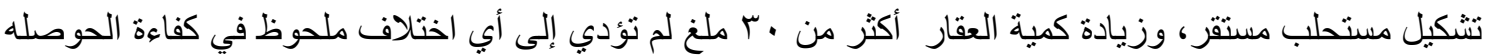

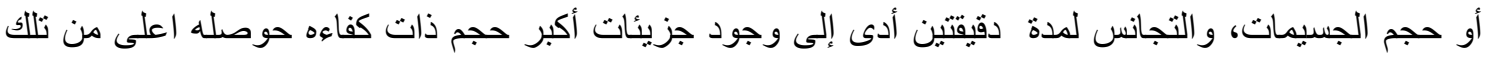

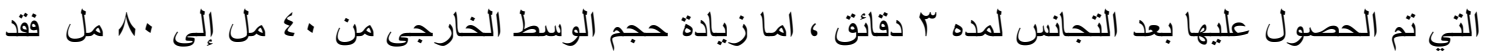

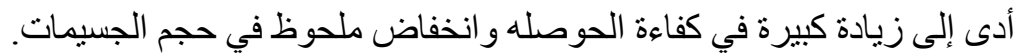

